\title{
镍催化烷基羧酸与对甲苯磺酸甲酯还原偶联甲基化成酮反应
}

\author{
顾 君 ${ }^{a}$ 刘建东 ${ }^{b}$ 孙雨人 ${ }^{a}$ 王洪宇 $*, b$ \\ $\left({ }^{a}\right.$ 上海大学材料科学与工程学院 上海 200444) \\ $\left({ }^{b}\right.$ 上海大学理学院化学系 上海 200444)
}

\begin{abstract}
摘要 甲基是最小的含碳取代基团, 在化合物中起到重要的作用. 羧酸转化为酮是有机化学的基础反应, 这种官能团 变化通常是间接地将羧酸先转化为酰氯或者 Weinreb 酰胺再由有机金属试剂进行亲核进攻. 直接将羧酸转变为酮, 需 要至少 2 equiv. 锂试剂, 同时反应需要在低温下进行, 往往伴随着很多叔醇的副产物. 报道了利用镍催化还原偶联的方 法, 将有机羧酸与对甲苯磺酸甲酯进行还原偶联, 一步反应直接由羧酸甲基化成酮, 其特点是原料易得, 反应条件温 和, 可以得到中等的收率并且有较好的底物适应性.
\end{abstract}

关键词 甲基化; $\mathrm{Ni}$ 催化; 芳香羧酸; 还原偶联

\section{Nickel-Catalyzed Reductive Methylation of Alkyl Acid with Methyl $p$-Tosylate}

\author{
Gu, Jun ${ }^{a} \quad$ Liu, Jiandong ${ }^{a} \quad$ Sun, Yuren ${ }^{b}$ Wang, Hongyu ${ }^{*, b}$ \\ ( ${ }^{a}$ School of Materials Science and Engineering, Shanghai University, Shanghai 200444) \\ ( ${ }^{b}$ Department of Chemistry, Shanghai University, Shanghai 200444)
}

\begin{abstract}
Methyl group is the smallest carbon substituent that plays important roles in many compounds. Conversion of a carboxylic acid into a ketone is a fundamental transformation in organic chemistry. This functional group change is usually performed indirectly by activating the carboxylic acid into an acyl chloride or into a Weinreb amide, and then by nucleophilic attack with an organometallic reagent. However, direct conversion of a carboxylic acid into a ketone can be achieved in one-step reaction using at least 2 equiv. of an organolithium reagent at low temperature and producing much tertiary alcohol. Herein, methylation of alkyl acid under Ni-catalyzed reductive coupling conditions using methyl $p$-methyl tosylate as the methylation reagent was reported to yield methylated ketones. Moderate yields as well as good functional group tolerance were observed under the present mild and easy-to-operate reaction conditions.

Keywords methylation; nickel-catalyzed; alkyl acid; reductive coupling
\end{abstract}

甲基或氟甲基 ${ }^{[15]}$ 是有机化合物中常见的官能团， 甲基化或氟甲基化是一类重要的反应，化合物中引入甲 基或氟甲基可以极大地促进分子油溶性 ${ }^{[6]}$, 所以在药物 分子 ${ }^{[7]}$ 以及材料中常常被引入 ${ }^{[8]}$ 来改变物质的性质. 酮 类化合物是有机化学中一类重要化合物, 将羧酸转换为 酮是有机化学中一类既重要又基础的反应 ${ }^{[9 \sim 12]}$. 常见的

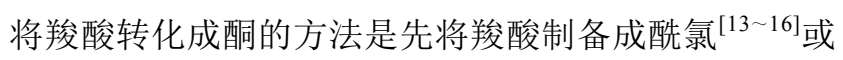
者 Weinreb 酰胺, 以此来对羧酸进行活化 ${ }^{[17 ~ 19]}$, 然后利 用有机金属试剂(比如有机锂试剂、格氏试剂等)进行亲 核反应来获取相对应的酮类化合物. 这类方法可以将羧
酸转化成对应的酮, 并且反应高效, 副产物极少, 劣势 在于整个转化过程需要经历多步反应. 利用有机锂试剂 可以直接一步将羒酸制备成酮, 但是这个方法也具有明 显的缺陷: (1)容易形成叔醇副产物; (2)官能团容忍性低; (3) 反应条件较为苛刻, 需要极低的温度 ${ }^{[20 \sim 25]}$.

近年来, 利用 $\mathrm{Ni}$ 催化还原偶联 ${ }^{[26]}$ 将羧酸转化成酮 的反应受到广泛的关注. Gosmini 等 ${ }^{[27]}$ 在 2003 年报道了 $\mathrm{CoBr}_{2}$ 和 $\mathrm{ZnBr}_{2}$ 催化下芳香溴化物和酰氯反应生成芳香 酮的反应, 这个反应体系需要在原位生成有机锌, 然后 有机锌再参与进反应循环体系, 操作过程较为复杂, 收

* Corresponding author. E-mail: wanghy@shu.edu.cn

Received March 24, 2017; revised April 16, 2017; published online May 17, 2017.

Project supported by the National Natural Science Foundation of China (No. 61204020) and the Innovation Program of Shanghai Municipal Education Commission (No. 15ZZ047).

国家自然科学基金(No. 61204020)和上海市教委创新(No. 15ZZ047)资助项目. 
率也不高. 郑建鸿等 ${ }^{[28]}$ 报道了利用芳香碘代物在 $\mathrm{Ni} / \mathrm{Zn}$ 条件下还原偶联得到酰胺或者亚胺类化合物. 2012 年, Weix 课题组 ${ }^{[29]}$ 成功实现了在 $\mathrm{Ni}$ 催化下, 由 $\mathrm{Mn}$ 或者 $\mathrm{Zn}$ 作还原剂, 烷基酰氯与烷基卤代物进行交叉偶联反应生 成酮. 与此同时我们课题组也报道了 $\mathrm{Ni}$ 催化下烷基卤 代物与酸及其衍生物 (如酰氯 ${ }^{[30]}$ 、芳香酸酐 ${ }^{[31]}$ 及酸 ${ }^{[32]}$ )的 羰基化反应, 实现了交叉偶联成酮的方法, 其中烷基碘 代物与酰氯的反应放大到克级后产率基本保持一致 ${ }^{[33]}$. 我们还进一步将 $\mathrm{MeOTs}$ 作为甲基亲电试剂, 实现了芳 香酸甲基化生成甲基酮的新方法 ${ }^{[34]}$. 这也是首次在文 献中将非常活泼的甲基亲电试剂用于还原偶联的报道. 但该方面的工作仅限于芳香酸底物, 对烷基酸效率很 低, 化合物 2 在该条件下仅有 $35 \%$ 的收率. 这是因为烷 基酸与芳香酸中烷基和芳香基的微弱电性差异导致他 们的反应活性的差异. 例如, 我们在对芳香酸 ${ }^{[2]}$ 与卤代 烷烃 ${ }^{[35]}$ 的酮化反应时所采用的条件与烷基酸有较大的 不同. 本论文实现了新的 $\mathrm{Ni}$ 催化还原偶联条件, 在课题 组先前陆文涁工作 ${ }^{[33]}$ 的基础上, 采用原位形成的缓和 酸䣶代替酰氯进行反应, 将混合溶剂改为四氢呋喃 (THF), 使用位阻更大的配体(化合物 3a) 代替化合物 3d, 一步将烷基羧酸甲基化形成相对应的酮类化合物(Eq. 1). 本方法条件温和, 底物适应性好, 有望为现有的甲 基酮制备方法提供一个重要的补充.

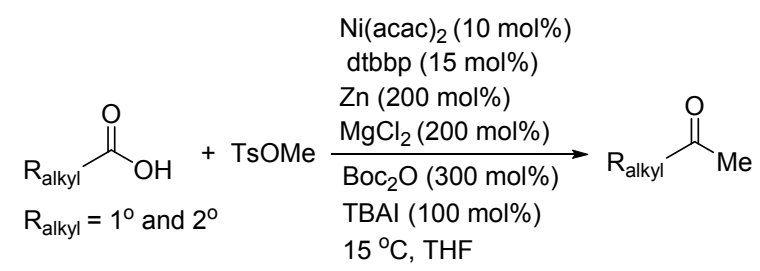

\section{1 结果与讨论}

\section{1 条件篮选}

选用 1 equiv. 3-苯基丙酸(1)作为模型反应底物, 1.5 equiv. 对甲苯磺酸甲酯作为甲基源进行反应的篮选(表 1). 首先, 对于催化剂 $\mathrm{Ni}$ 源进行篮选. 选用 $\mathrm{NiBr}_{2} \bullet$ diglyme 作催化剂时, 收率较低, 只有 $41 \%$ (Entry 1). 使用其他一些常见的 $\mathrm{Ni}$ 催化剂[如 $\mathrm{Ni}(\mathrm{cod})_{2}$, $\mathrm{NiI}_{2}, \mathrm{NiBr}_{2}$ ] 都不如 $\mathrm{Ni}(\mathrm{acac})_{2}$ 的收率好. 因此, $\mathrm{Ni}$ 源使用 $\mathrm{Ni}(\mathrm{acac})_{2}$ 来作优化催化剂. 接着对配体进行篮选. 位 阻较大的二联吡啶类配体(3a)优于位阻较小的(3b). 三 齿配体(4)与卡宾配体(5)效果都不理想, 收率下降甚至 只有痕量的收率(图 1). 可能二齿配体(3)在这个反应中 与催化剂 $\mathrm{Ni}(\mathrm{acac})_{2}$ 络合效果更好. 随后对于溶剂进行 篎选. 选用 DMSO (Entry 11)、DMA (Entry 12)和乙腈 (Entry 13), 反应的收率都不理想, 因此选择 THF 作为
最佳反应溶剂. 最后，对添加剂进行篮选. 反应中去除 TBAI (Entry 14), 几乎没有收率; 如果不加入 $\mathrm{Boc}_{2} \mathrm{O}$ (Entry 15), 则完全观察不到产物. 在我们课题组 ${ }^{[32]}$ 先 前的工作中发现, $\mathrm{Boc}_{2} \mathrm{O}$ 和 $\mathrm{MgCl}_{2}$ 在以羧酸为底物的反 应中，可以促进羧酸底物形成酸䣶中间体，然后进一 步与对甲苯磺酸甲酯进行还原偶联. 因此, 经过篮选 最优的标准条件为(Entry 5): $10 \mathrm{~mol} \% \mathrm{Ni}(\mathrm{acac})_{2}$ 为催化 剂, $15 \mathrm{~mol} \% \mathrm{dtbbp} \mathrm{(3)}$ 为配体, $200 \mathrm{~mol} \%$ 锌粉作为还原 剂, $200 \mathrm{~mol} \% \mathrm{MgCl}_{2}, 300 \mathrm{~mol} \%$. $\mathrm{Boc}_{2} \mathrm{O}, 100 \mathrm{~mol} \%$ TBAI 作为添加剂, $1 \mathrm{~mL}$ THF 作为溶剂, 在 $15{ }^{\circ} \mathrm{C}$ 或室 温下反应 $12 \mathrm{~h}$.

表 1 反应条件的优化

Table 1 Optimization of reaction conditions

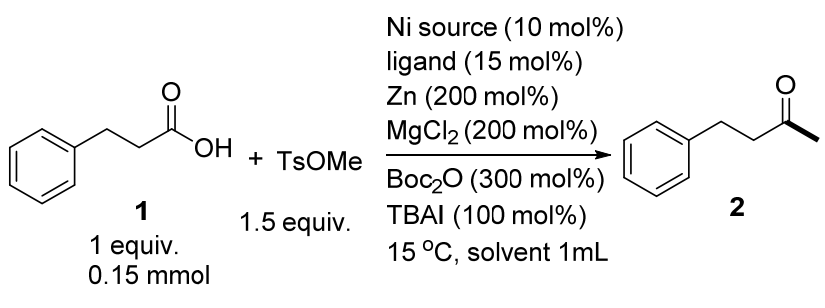

\begin{tabular}{|c|c|c|c|c|c|}
\hline Entry & Cat. & Ligand & Solvent & Additive & Yield $^{a} \%$ \\
\hline 1 & $\mathrm{NiBr}_{2} \bullet$ diglyme & $3 \mathbf{a}$ & THF & TBAI & 41 \\
\hline 2 & $\mathrm{Ni}(\operatorname{cod})_{2}$ & $3 \mathbf{a}$ & THF & TBAI & 10 \\
\hline 3 & $\mathrm{NiI}_{2}$ & $3 \mathbf{a}$ & THF & TBAI & 6 \\
\hline 4 & $\mathrm{NiBr}_{2}$ & 3a & THF & TBAI & 32 \\
\hline 5 & $\mathrm{Ni}(\mathrm{acac})_{2}$ & $3 \mathbf{a}$ & THF & TBAI & 55 \\
\hline 6 & $\mathrm{Ni}(\text { acac })_{2}$ & $3 \mathbf{b}$ & THF & TBAI & 33 \\
\hline 7 & $\mathrm{Ni}(\mathrm{acac})_{2}$ & $3 c$ & THF & TBAI & 39 \\
\hline 8 & $\mathrm{Ni}(\mathrm{acac})_{2}$ & 3d & THF & TBAI & 26 \\
\hline 9 & $\mathrm{Ni}(\mathrm{acac})_{2}$ & 4 & THF & TBAI & 25 \\
\hline $10^{b}$ & $\mathrm{Ni}(\text { acac })_{2}$ & 5 & THF & TBAI & Trace \\
\hline 11 & $\mathrm{Ni}(\mathrm{acac})_{2}$ & $3 \mathbf{a}$ & $\mathrm{MeCN}$ & TBAI & 45 \\
\hline 12 & $\mathrm{Ni}(\mathrm{acac})_{2}$ & $3 \mathbf{a}$ & DMA & TBAI & 20 \\
\hline 13 & $\mathrm{Ni}(\mathrm{acac})_{2}$ & $3 \mathbf{a}$ & DMSO & TBAI & Trace \\
\hline 14 & $\mathrm{Ni}(\mathrm{acac})_{2}$ & $3 \mathbf{a}$ & THF & No TBAI & Trace \\
\hline 15 & $\mathrm{Ni}(\text { acac })_{2}$ & $3 \mathbf{a}$ & THF & No $\mathrm{Boc}_{2} \mathrm{O}$ & N.D. \\
\hline 16 & $\mathrm{Ni}(\mathrm{acac})_{2}$ & $3 \mathbf{a}$ & THF & No $\mathrm{MgCl}_{2}$ & 20 \\
\hline
\end{tabular}

${ }^{a}$ Yields were determined by ${ }^{1} \mathrm{H}$ NMR with 2,5-dimethylfuran as an internal standard (calibrated); ${ }^{b} 100 \mathrm{~mol} \%$ pyridine as base.<smiles>[R]c1cnc(-c2cc([R])c([R])cn2)cc1[R]</smiles><smiles>CC(C)C1COC(c2cccc(C3=N[C@@H](C(C)C)CO3)n2)=N1</smiles>

3a: $R^{1}=t B u, R^{2}=H$ 3b: $R^{1}=H, R^{2}=H$ 3c: $R^{1}=M e, R^{2}=H$ 3d: $R^{1}=H, R^{2}=M e$

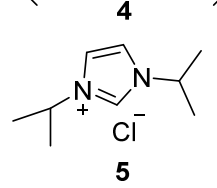

图 1 配体 $\mathbf{3} \sim 5$ 的结构

Figure 1 Structures of ligands $3 \sim 5$ 


\section{2 底物扩展}

通过模板反应篮选出反应最佳条件(Eq. 2), 对反应 底物进行普适性考察. 以 3-苯基丙酸(1)为原料, 作为标 准反应物, 反应获得苄基丙酮(2), 可以达到 55\%的收 率. 在羧酸上增加一个碳原子后, 以 3 -苯基丁酸为底物, 化合物 6 也能够获得 55\%的收率，与模板反应相似. 接 下来选择改变苯环上的取代基来研究标准条件下底物 的适应性. 可以看到在苯环对位引入甲基(12)以及甲氧 基(13)后，对应产率有少量提高. 对位取代基为氯(14) 时收率也略微提高, 改为氟(10)时, 收率变化不大, 对 位取代基替换三氟甲基时(7), 相对应产物收率有明显 降低. 总体来说, 对于一级烷基酸而言, 这个标准条件 的适应性较好. 但是对于二级烷基酸进行尝试, 发现二 级烷基酸 $(8,11$ 和 15$)$ 的收率相比一级烷基酸有明显降 低. 遗憾的是, Boc 保护的氨基酸(17 和 18)在该条件下 无法检测到任何产物, 可能含 $\mathrm{N}$ 化合物容易和催化剂络 合，导致无法发生反应.

表 2 烷基酸与对甲苯磺酸甲酯成酮底物扩展

Table 2 Scope of alkyl acid with methyl $p$-tosylate<smiles>CC(=O)CCc1ccccc1</smiles>

Yield: $55 \%^{a}$

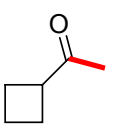

8

Yield: $30 \%^{b}$<smiles>CC(=O)C1CCCCC1</smiles>

11

Yield: $35 \%^{b}$

14

Yield: $63 \%^{a}$<smiles>CC(=O)C1Cc2ccccc2N1C(=O)OC(C)(C)C</smiles>

17

N.D.<smiles>CC(=O)CCc1ccc(Cl)cc1</smiles><smiles>CCCCCc1ccccc1</smiles>

9

Yield: $53 \%^{a}$<smiles>CC(=O)CCc1ccc(C)cc1</smiles>

12

Yield: $61 \%$

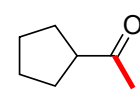

15

Yield: $30 \%^{b}$

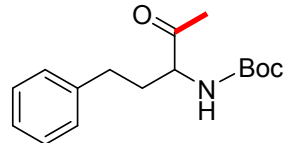

18

N.D.

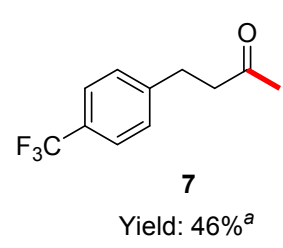<smiles>CC(=O)CCc1ccc(F)cc1</smiles>

10

Yield: $53 \%^{a}$<smiles>COc1ccc(CCC(C)=O)cc1</smiles>

13 Yield: $62 \%^{a}$<smiles>CC(=O)CCC(c1ccccc1)c1ccccc1</smiles>

16

Yield: $61 \%^{a}$

\footnotetext{
${ }^{a}$ Yield of isolated product. ${ }^{b}$ Yield determined by ${ }^{1} \mathrm{H}$ NMR spectroscopy using

2,5-dimethylfuran as the internal reference.
}

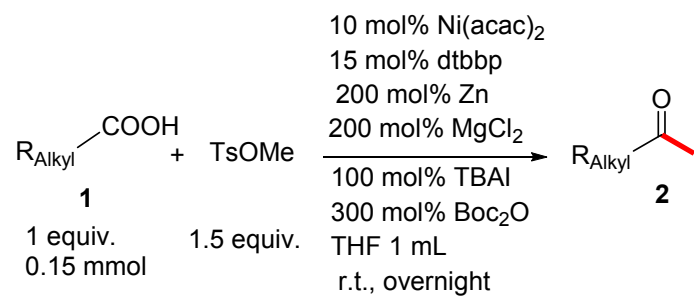

\section{3 可能的反应机理}

$\mathrm{Ni}$ 催化还原偶联反应的机理较为复杂, 我们对这 个反应的机理进行推测. MeOTs 可能会与反应中碘离子 (四丁基碘化铵)或氯离子(氯化镁)交换缓慢生成较稀浓 度的碘甲烷或者氯甲烷参与反应，由于生成 $\mathrm{MeX}$ 浓度 较稀，降低了 $\mathrm{MeX}$ 与镍氧化加成的速率，提高了反应选 择性 ${ }^{[34]}$.二价 $\mathrm{Ni}$ 中间体 $\mathbf{I}$ 与甲基自由基发生氧化加成生 成三价 $\mathrm{Ni}$ 中间体 II, 三价 $\mathrm{Ni}$ 中间体经历还原消除得到 甲基酮产物和一价 $\mathrm{Ni}$ 中间体 III，一价 $\mathrm{Ni}$ 中间体与 $\mathrm{MeX}$ 作用释放出甲基自由基和二价 $\mathrm{Ni}$ 中间体 $\mathbf{I V}$ ，最后二价 $\mathrm{Ni}$ 中间体 IV 被锌粉还原为零价 $\mathrm{Ni}$ 中间体完成催化循 环(Scheme 1).

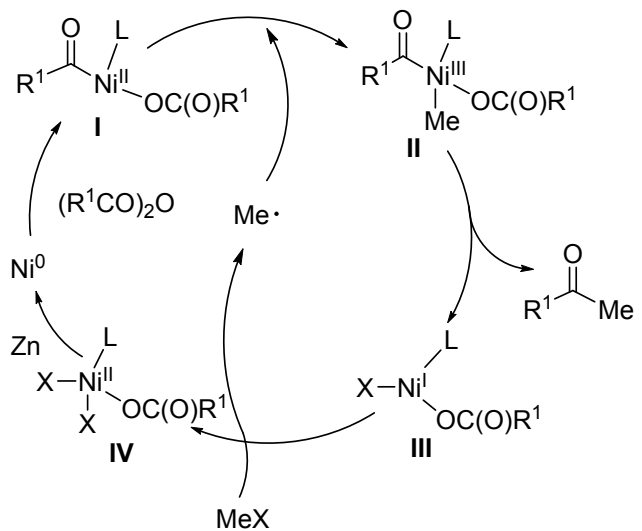

图式 1 与对甲苯磺酸甲酯甲基化成酮的可能机理

Scheme 1 Proposed catalytic cycle for methylation of methyl $p$-tosylate

\section{2 结论}

本论文发展了催化成甲基酮的新方法，利用金属 $\mathrm{Ni}$ 作为催化剂一步将烷基羧酸甲基化成酮，获得中等 收率, 并且底物的适应性较好, 可以容忍较多种不同烷 基酸底物. 与传统羧酸甲基化转化成酮的方法相比，克 服了反应条件苛刻，副产物多，步骤繁琐等缺陷．本方 法为现有的成酮反应，特别是烷基甲基酮的制备提供了 一个新的补充.

\section{3 实验部分}

\section{1 仪器与试剂}

核磁数据均由 Bruker $500 \mathrm{MHz}$ 核磁共振仪分析, 
氛代氯仿在 ${ }^{1} \mathrm{H}$ NMR 和 ${ }^{13} \mathrm{C}$ NMR 谱图中的化学位移分 别定于 $\delta 7.26$ 和 77.0. 高分辨质谱(HRMS)数据由 Bruker APEXIII 7.0 和 IonSpec 4.7 TESLA FTMS 分析所得到; 低分辨质谱数据由岛津公司的 GCMS-QP2010 仪器测试 所得.

除非有特别说明, 所有的反应都在氮气条件下操 作. 无水 THF 是从钠/二苯甲酮体系中重蒸得到. 其它 使用的反应溶剂都是分析纯的. Zn $(99.9 \%$, powder, 阿 拉丁), $\mathrm{NiI}_{2}$ (99.5\%, Alaf Aesar), Ni(cod) 2 (99\%, Stream), $\mathrm{NiBr}_{2}$ (99.5\%, Alaf Aesar), Ni(acac) 2 (99\%, Alaf Aesar), $\mathrm{NiBr}_{2} \bullet$ diglyme (99.5\%, Strem). $\mathrm{N}, \mathrm{N}$-二甲基乙酰胺 DMA (99.8\%, SuperDry, with molecular sieves), 二甲基亚砜 DMSO (99.8\%, SuperDry, with molecular sieves), 乙腈 $\mathrm{CH}_{3} \mathrm{CN}$ (99.5\%, SuperDry, with molecular sieves). 其余 反应底物试剂均购自安耐吉、百灵威、伊诺凯等公司. 柱 层析色谱柱使用 300 至 400 目硅胶(购于青岛海洋).

\section{2 实验方法与数据}

还原甲基化成酮反应标准操作：向装有摚拌子并在 煤气灯上干燥过的 Schlenk 反应瓶中加入羧酸底物 ( 0.15 $\mathrm{mmol}, 100 \mathrm{~mol} \%)$ 、配体 3 (0.0225 mmol, $15 \mathrm{~mol} \%)$ 、 MeOTs $(0.225 \mathrm{mmol}, 150 \%)$ 、锌粉 $(0.3 \mathrm{mmol}, 200 \mathrm{~mol} \%)$. Schlenk 反应瓶转移到手套箱内, 在 $30 \mathrm{~min}$ 内换气 3 次, 然后在手套箱内加入 $\mathrm{Ni}(\mathrm{acac})_{2}(0.015 \mathrm{mmol}, 10 \mathrm{~mol} \%)$ 和 氯化镁 $(0.3 \mathrm{mmol}, 200 \mathrm{~mol} \%)$. Schlenk 反应瓶被移出手 套箱, $\mathrm{Boc}_{2} \mathrm{O}(0.45 \mathrm{mmol}, 300 \mathrm{~mol} \%)$ 和 THF $(1 \mathrm{~mL})$ 通过 注射器加入. 反应体系在 $15{ }^{\circ} \mathrm{C}$ 下反应 $12 \mathrm{~h}$, 反应结束 之后直接上层析硅胶柱分离产物, 得到相应的液体产 物.

芐基丙酮(2): 无色油状液体， $12.2 \mathrm{mg}$, 产率 55\%. ${ }^{1} \mathrm{H}$ NMR $\left(500 \mathrm{MHz}, \mathrm{CDCl}_{3}\right) \delta: 7.33 \sim 7.29(\mathrm{~m}, 3 \mathrm{H})$, $7.24 \sim 7.21(\mathrm{~m}, 2 \mathrm{H}), 2.93(\mathrm{t}, J=7.4 \mathrm{~Hz}, 2 \mathrm{H}), 2.78(\mathrm{t}, J=$ $7.4 \mathrm{~Hz}, 2 \mathrm{H}), 2.16(\mathrm{~s}, 3 \mathrm{H}) ;{ }^{13} \mathrm{C} \mathrm{NMR}\left(125 \mathrm{MHz}, \mathrm{CDCl}_{3}\right) \delta$ : 207.9, 141.1, 128.5, 128.3, 126.1, 45.1, 30.1, 29.7; HRMS (ESI) calcd for $\mathrm{C}_{10} \mathrm{H}_{12} \mathrm{O}: 148.0888$, found 148.0891 .

4-芐基-2-丁酮(6): 无色液体, $24.3 \mathrm{mg}$, 产率 55\%. ${ }^{1} \mathrm{H}$ NMR $\left(500 \mathrm{MHz}, \mathrm{CDCl}_{3}\right) \delta: 7.32 \sim 7.28(\mathrm{~m}, 2 \mathrm{H})$, $7.22 \sim 7.19(\mathrm{~m}, 2 \mathrm{H}), 2.65(\mathrm{t}, J=7.6 \mathrm{~Hz}, 2 \mathrm{H}), 2.45(\mathrm{t}, J=$ $7.6 \mathrm{~Hz}, 2 \mathrm{H}), 2.14$ (s, 3H), $1.96 \sim 1.91(\mathrm{~m}, 2 \mathrm{H}) ;{ }^{13} \mathrm{C}$ NMR $\left(125 \mathrm{MHz}, \mathrm{CDCl}_{3}\right) \delta: 208.8,141.5,128.5,128.3,125.9$, 42.8, 35.1, 29.9, 25.2; HRMS (ESI) calcd for $\mathrm{C}_{11} \mathrm{H}_{14} \mathrm{O}$ : 162.1045, found 162.1047 .

(4-三氟甲基苄基)丙酮(7): 无色液体，14.9 mg, 产 率 46\%. ${ }^{1} \mathrm{H}$ NMR $\left(500 \mathrm{MHz}, \mathrm{CDCl}_{3}\right) \delta: 7.55$ (d, $J=8.1$ $\mathrm{Hz}, 2 \mathrm{H}), 7.32$ (d, $J=8.1 \mathrm{~Hz}, 2 \mathrm{H}), 2.96(\mathrm{t}, J=7.4 \mathrm{~Hz}, 2 \mathrm{H})$, $2.80(\mathrm{t}, J=7.4 \mathrm{~Hz}, 2 \mathrm{H}), 2.17$ (s, 3H); ${ }^{19} \mathrm{~F}$ NMR $(500 \mathrm{MHz}$,
$\left.\mathrm{CDCl}_{3}\right) \delta:-62.39 ;{ }^{13} \mathrm{C} \mathrm{NMR}\left(125 \mathrm{MHz}, \mathrm{CDCl}_{3}\right) \delta: 207.1$, 145.1, 128.6 (q, $J=37.4 \mathrm{~Hz}), 125.4(\mathrm{q}, J=3.7 \mathrm{~Hz}), 123.1$ (q, $J=276.5 \mathrm{~Hz}$ ), 44.5, 30.1, 29.3; HRMS (ESI) calcd for $\mathrm{C}_{11} \mathrm{H}_{11} \mathrm{~F}_{3} \mathrm{O}: 216.0762$, found 216.0764.

环丁基甲基酩(8): $4.4 \mathrm{mg}$, 无色液体，产率 30\%. ${ }^{1} \mathrm{H}$ NMR $\left(500 \mathrm{MHz}, \mathrm{CDCl}_{3}\right) \delta: 3.26 \sim 3.21(\mathrm{~m}, 1 \mathrm{H}), 2.22 \sim$ $2.08(\mathrm{~m}, 4 \mathrm{H}), 2.04(\mathrm{~s}, 3 \mathrm{H}), 2.04 \sim 1.91(\mathrm{~m}, 1 \mathrm{H}), 1.81 \sim$ $1.76(\mathrm{~m}, 1 \mathrm{H}) ;{ }^{13} \mathrm{C}$ NMR $\left(125 \mathrm{MHz}, \mathrm{CDCl}_{3}\right) \delta: 209.9,46.1$, 26.9, 24.2, 17.5; HRMS (ESI) calcd for $\mathrm{C}_{6} \mathrm{H}_{10} \mathrm{O}: 98.0732$, found 98.0734

(2-甲氧基苄基)丙酮(9): 无色液体，14.2 $\mathrm{mg}$, 产率 53\%. ${ }^{1} \mathrm{H}$ NMR (500 MHz, $\left.\mathrm{CDCl}_{3}\right) \delta: 7.23 \sim 7.15(\mathrm{~m}, 2 \mathrm{H})$, $6.92 \sim 6.86(\mathrm{~m}, 3 \mathrm{H}), 3.84(\mathrm{~s}, 3 \mathrm{H}), 2.91(\mathrm{t}, J=8.0 \mathrm{~Hz}, 2 \mathrm{H})$, $2.76(\mathrm{t}, J=8.0 \mathrm{~Hz}, 2 \mathrm{H}), 2.16$ (s, 3H); ${ }^{13} \mathrm{C}$ NMR $(125 \mathrm{MHz}$, $\left.\mathrm{CDCl}_{3}\right) \delta: 208.6,157.4,129.9,129.2,127.4,120.4,110.2$, 55.1, 43.7, 29.9, 25.1; HRMS (ESI) calcd for $\mathrm{C}_{11} \mathrm{H}_{14} \mathrm{O}_{2}$ : 178.0994, found 178.1008 .

(4-氟苄基)丙酮(10): 无色液体, $13.2 \mathrm{mg}$, 产率 53\%. ${ }^{1} \mathrm{H}$ NMR $\left(500 \mathrm{MHz}, \mathrm{CDCl}_{3}\right) \delta: 7.16(\mathrm{~d}, J=8.0 \mathrm{~Hz}, 2 \mathrm{H})$, $6.98(\mathrm{~d}, J=8.0 \mathrm{~Hz}, 2 \mathrm{H}), 2.90(\mathrm{t}, J=7.4 \mathrm{~Hz}, 2 \mathrm{H}), 2.76(\mathrm{t}$, $J=7.4 \mathrm{~Hz}, 2 \mathrm{H}), 2.16(\mathrm{~s}, 3 \mathrm{H}) ;{ }^{19} \mathrm{~F}$ NMR $\left(500 \mathrm{MHz}, \mathrm{CDCl}_{3}\right)$ $\delta:-117.31(\mathrm{~m}) ;{ }^{13} \mathrm{C}$ NMR $\left(125 \mathrm{MHz}, \mathrm{CDCl}_{3}\right) \delta: 207.6$, $162.3(\mathrm{~d}, J=244.8 \mathrm{~Hz}), 136.6(\mathrm{~d}, J=3.1 \mathrm{~Hz}), 129.7(\mathrm{~d}$, $J=17.8 \mathrm{~Hz}), 115.3(\mathrm{~d}, J=21.3 \mathrm{~Hz}), 45.2,30.1,29.7$; HRMS (ESI) calcd for $\mathrm{C}_{10} \mathrm{H}_{11} \mathrm{FO}$ : 166.0794, found 167.0833 .

甲基酮环己酯(11): 无色液体， $6.6 \mathrm{mg}$, 产率 $35 \%$. ${ }^{1} \mathrm{H}$ NMR $\left(500 \mathrm{MHz}, \mathrm{CDCl}_{3}\right) \delta: 2.35 \sim 2.31(\mathrm{~m}, 1 \mathrm{H}), 2.13$ $(\mathrm{s}, 3 \mathrm{H}), 1.89 \sim 1.86(\mathrm{~m}, 2 \mathrm{H}), 1.81 \sim 1.76(\mathrm{~m}, 2 \mathrm{H}), 1.69 \sim$ $1.65(\mathrm{~m}, 1 \mathrm{H}), 1.36 \sim 1.17(\mathrm{~m}, 5 \mathrm{H}) ;{ }^{13} \mathrm{C}$ NMR $(125 \mathrm{MHz}$, $\mathrm{CDCl}_{3}$ ) $\delta: 212.3,51.4,28.4,27.8,25.8,25.6$; HRMS (ESI) calcd for $\mathrm{C}_{8} \mathrm{H}_{14} \mathrm{O}: 126.1045$, found 126.1048 .

(4-甲基苄基)丙酩 ${ }^{[36]}(\mathbf{1 2})$ : 无色液体, $14.8 \mathrm{mg}$, 产率 61\%. ${ }^{1} \mathrm{H}$ NMR $\left(500 \mathrm{MHz}, \mathrm{CDCl}_{3}\right) \delta: 7.12 \sim 7.07(\mathrm{~m}, 4 \mathrm{H})$, $2.86(\mathrm{t}, J=7.0 \mathrm{~Hz}, 2 \mathrm{H}), 2.75(\mathrm{t}, J=7.0 \mathrm{~Hz}, 2 \mathrm{H}), 2.31(\mathrm{~s}$, $3 \mathrm{H}), 2.13(\mathrm{~s}, 3 \mathrm{H}) ;{ }^{13} \mathrm{C}$ NMR (125 MHz, $\left.\mathrm{CDCl}_{3}\right) \delta: 207.1$, 137.9, 135.7, 129.3, 128.2, 45.4, 30.1, 29.4, 21.1; HRMS (ESI) calcd for $\mathrm{C}_{11} \mathrm{H}_{14} \mathrm{O}: 162.1045$, found 162.1049 .

(4-甲氧基芐基)丙酥(13): 无色液体, $16.5 \mathrm{mg}$, 收率 $62 \%$. ${ }^{1} \mathrm{H}$ NMR $\left(500 \mathrm{MHz}, \mathrm{CDCl}_{3}\right) \delta: 7.11(\mathrm{~d}, J=8.6 \mathrm{~Hz}$, $2 \mathrm{H}), 6.85$ (d, $J=8.6 \mathrm{~Hz}, 2 \mathrm{H}), 3.81$ (s, 3H), 2.86 (t, $J=7.0$ $\mathrm{Hz}, 2 \mathrm{H}), 2.74$ (t, $J=7.0 \mathrm{~Hz}, 2 \mathrm{H}), 2.15$ (s, 3H); ${ }^{13} \mathrm{C}$ NMR $\left(125 \mathrm{MHz}, \mathrm{CDCl}_{3}\right) \delta: 208.1,157.9,133.1,129.2,113.9$, 55.2, 45.4, 30.1, 28.9; HRMS (ESI) calcd for $\mathrm{C}_{11} \mathrm{H}_{14} \mathrm{O}_{2}$ : 178.0994, found 178.0996 . 
(4-氯苄基)丙酮(14): 无色液体, $17.2 \mathrm{mg}$, 收率 63\%. ${ }^{1} \mathrm{H}$ NMR $\left(500 \mathrm{MHz}, \mathrm{CDCl}_{3}\right) \delta: 7.26(\mathrm{~d}, J=8.2 \mathrm{~Hz}, 2 \mathrm{H})$, $7.14(\mathrm{~d}, J=8.2 \mathrm{~Hz}, 1 \mathrm{H}), 2.87$ (t, $J=7.3 \mathrm{~Hz}, 2 \mathrm{H}), 2.76$ (t, $J=7.3 \mathrm{~Hz}, 2 \mathrm{H}), 2.15$ (s, 3H); ${ }^{13} \mathrm{C} \mathrm{NMR}\left(125 \mathrm{MHz}, \mathrm{CDCl}_{3}\right)$ $\delta$ : 207.5, 139.4, 131.8, 129.7, 128.5, 44.9, 30.1, 28.9; HRMS (ESI) calcd for $\mathrm{C}_{10} \mathrm{H}_{11} \mathrm{ClO}$ : 182.0498, found 182.0477.

环戊基乙酮(15): 无色液体，5 mg, 收率 $30 \%$. ${ }^{1} \mathrm{H}$ NMR $\left(500 \mathrm{MHz}, \mathrm{CDCl}_{3}\right) \delta: 2.86 \sim 2.81(\mathrm{~m}, 1 \mathrm{H}), 2.12(\mathrm{~s}$, $3 \mathrm{H}), 1.83 \sim 1.52(\mathrm{~m}, 8 \mathrm{H}) ;{ }^{13} \mathrm{C}$ NMR $\left(125 \mathrm{MHz}, \mathrm{CDCl}_{3}\right) \delta$ : 211.2, 52.1, 28.6, 25.9; HRMS (ESI) calcd for $\mathrm{C}_{7} \mathrm{H}_{12} \mathrm{O}$ : 112.0888 , found 112.0891 .

5,5-二苯基-2-丁酮(16): 无色液体，21.8 mg，收率 61\%. ${ }^{1} \mathrm{H}$ NMR (500 MHz, $\left.\mathrm{CDCl}_{3}\right) \delta: 7.32 \sim 7.21(\mathrm{~m}, 10 \mathrm{H})$, 4.64 (t, $J=7.5 \mathrm{~Hz}, 1 \mathrm{H}), 3.22$ (d, $J=7.5 \mathrm{~Hz}, 2 \mathrm{H}), 2.11$ (s, $3 \mathrm{H}) ;{ }^{13} \mathrm{C}$ NMR (125 MHz, $\left.\mathrm{CDCl}_{3}\right) \delta: 206.9,143.8,128.6$, 127.7, 126.4, 49.6, 46.1, 30.6; HRMS (ESI) calcd for $\mathrm{C}_{17} \mathrm{H}_{18} \mathrm{O}: 238.1358$, found 238.1364 .

辅助材料(Supporting Information) 化合物 $2 \sim 16$ 的核 磁共振图谱. 这些材料可以免费从本刊网站(http://siocjournal. $\mathrm{cn} /$ )上下载.

\section{Referenes}

[1] Sheng, W.; Jin, C.; Shan, S.; Jia, Y.; Gao, J. Chin. J. Org. Chem. 2016, 36, 325 (in Chinese). (盛卫坚, 金城安, 单尚, 贾义霞, 高建荣, 有机化学, 2016, 36, 325.)

[2] Ni, C.; Zhu, L.; Hu, J. Acta Chim. Sinica 2015, 73, 90 (in Chinese). (倪传法, 朱林桂, 胡金波, 化学学报, 2015, 73, 90.)

[3] Gou, B.; Yang, C.; Zhang, L.; Xia, W. Acta Chim. Sinica 2017, 75, 66 (in Chinese) (苟宝权，杨超，张否，夏吾昫，化学学报, 2017, 75, 66.)

[4] Rong, J.; Ni, C.; Wang, Y.; Kuang, C.; Gu, Y.; Hu, J. Acta Chim. Sinica 2017, 75, 105 (in Chinese).

(荣健，倪传法，王云泽,劻翠文，顾玉诚，胡金波，化学学报, 2017, 75, 105.)

[5] Zhuang, H.; Zeng, R.; Zou, J. Chin. J. Org. Chem. 2016, 34, 368

[6] Heike, S.; Tim, C. Angew. Chem., Int. Ed. 2013, 52, 2.
[7] Barreiro, E. J.; Kümmerle, A. E.; Fraga, C. A. M. Chem. Rev. 2011, $111,5215$.

[8] Peretti, K. L.; Ajiro, H.; Cohen, C. T.; Lobkovsky, E. B.; Coates, G. W. J. Am. Chem. Soc. 2005, 127, 11566.

[9] Nicholson, J. W.; Wilson, A. D. J. Chem. Educ. 2004, 81, 1362.

[10] Wilkinson, M. C. Org. Lett. 2011, 13, 2232.

[11] Park, A.; Park, K.; Kim, Y.; Lee, S. Org. Lett. 2011, 13, 944.

[12] Gooßen, L.; Rodriguez, N.; Gooßen, K. Angew. Chem., Int. Ed. 2008, 47, 3100 .

[13] Posner, G. H.; Whitten, C. E.; McFarland, P. E. J. Am. Chem. Soc 1972, 94, 5106.

[14] Wang, X.; Zhang, L.; Sun, X.; Xu, Y.; Krishnamurthy, D.; Senanayake, C. H. Org. Lett. 2005, 7, 5593

[15] Ryu, I.; Ikebe, M.; Sonoda, N.; Yamato, S.; Yamamura, G.; Komatsu, M. Tetrahedron Lett. 2002, 43, 1257.

[16] Dieter, R. K. Tetrahedron 1999, 55, 4177.

[17] Nahm, S.; Weinreb, S. M. Tetrahedron Lett. 1981, 22, 3815.

[18] Kangani, C. O.; Kelley, D. E.; Day, B. W. Tetrahedron Lett. 2006, 6289.

[19] Qu, B.; Collum, D. B. J. Org. Chem. 2006, 71, 7117.

[20] Alonso, F.; Lorenzo, E.; Yus, M. J. Org. Chem. 1996, 61, 6058.

[21] Gilman, H.; van Ess, P. R. J. Am. Chem. Soc. 1933, 55, 1258.

[22] Tegner, C. Acta Chem. Scand 1952, 6, 782.

[23] Jorgenson, M. J. Org. React. 1970, 18, 1.

[24] Bare, T. M.; House, H. O. Org. Synth. 1973, 5, 775.

[25] Lubell, W. D.; Rapoport, H. J. Am. Chem. Soc. 1988, 110, 7447

[26] (a) Gu, J.; Wang, X.; Xue, W.; Gong, H. Org. Chem. Front. 2015, 2 , 1411.

(b) Everson, D. A.; Weix, D. J. J. Org. Chem. 2014, 79, 4793.

(c) Knappke, C. E.; Grupe, S.; Gärtner, D.; Corpet, M.; Gosmini, C.; Jacobi von Wangelin, A. Chem. Eur. J. 2014, 20, 6828.

(d) Weix, D. J. Acc. Chem. Res. 2015, 48, 1767.

(e) Moragas, T.; Correa, A.; Martin, R. Chem. Eur. J. 2014, 20, 8242 .

(f) Wang, X.; Dai, Y.; Gong, H. Top. Curr. Chem. 2016, 374, 43.

[27] Fillon, F.; Gosmini, C.; Perichon, J. Tetrahedron 2003, 59, 8199.

[28] Hsieh, J.-H.; Cheng, C.-H. Chem. Commun. 2005, 4554

[29] Wotal, A.; Weix, D. J. Org. Lett. 2012, 14, 1476.

[30] Wu, F.; Gong, H. Org. Lett. 2012, 14, 3044.

[31] Yin, H.; Zhao, C.; You, H.; Lin, Q.; Gong, H. Chem. Commun. 2012, 48, 7034 .

[32] Zhao, C.; Jia, X.; Wang, X.; Gong, H. J. Am. Chem. Soc. 2013, 136, 17645

[33] Lu, W.; Liang, Z.; Zhang, Y.; Wu, F.; Qian, Q.; Gong, H. Synthesis 2013, 45, 2234.

[34] Liang, Z.; Xue, W.; Lin, K.; Gong, H. Org. Lett. 2014, 16, 5620.

[35] Jia, X.; Zhang, X.; Qian, Q.; Gong, H. Chem. Commun. 2015, 51, 10302.

[36] Christelle, P.; François, C.; Bertrand, C. Org. Lett. 2001, 3, 803. 